\title{
MAPA PLUVIOMÉTRICO DE ESPAÑA PENINSULAR Y BALEARES \\ (EN EL PERÍODO INTERNACIONAL 1961-1990)
}

\author{
José Jaime Capel Molina
}

\section{RESUMEN}

Nuestro trabajo de Investigación es un comentario geográfico del mapa pluviométrico medio de España Peninsular y Baleares (en el período internacional 1961-1990). El mapa ha sido trazado a escala 1:1.000.000, con los datos de 689 estaciones pluviométricas. La pluviometría española presenta cifras muy desiguales en función a lo compartimentado de su relieve. Así entre el N/NW y el SE peninsular se producen los contrastes más acusados provocando el máximo gradiente vegetal y pluviométrico. A lo largo del meridiano $2^{\circ}$ Longitud Oeste, se origina el máximo gradiente, desde el observatorio de Articutza (Navarra Atlántica), a 43ำ $12^{\prime} \mathrm{N}$, con $2.661 \mathrm{~mm}$., hasta los $160 \mathrm{~mm}$. de cabo de Gata (Almería), a $36^{\circ} 45^{\prime}$.

Palabras clave: Clima, Pluviometría, Isoyetas, España peninsular y Baleares.

\section{RÉSUMÉ}

Carte pluviométrique de l'Espagne péninsulaire et des Îles Baléares (Période internationale 1961-1990).

Notre travail de recherche est un commentaire géographique de la carte pluviométrique moyenne de l'Espagne péninsulaire et des îles Baléares. (Pendant la période internationale qui va de 1961 à 1990). La carte a été dressée à l'echelle 1: 1.000.000, avec les données de 689 stations pluviométriques. La pluviométrie espagnole présente des chiffres bien inégaux par rapport au relief du pays. Ainsi, entre le Nord et le Sud-Est péninsulaire on se trouve face aux plus accusés contrastes, ce qui provoque le maximun de gradient végétal et pluviométrique. Le long du méridien $2^{\circ} \mathrm{L}$.W, on origine le gradient maximal de l'observatoire de Articutza (la Navarre atlantique) à $43^{\circ} 12^{\prime} \mathrm{N}$, avec 2661 mm., aux 160 mm. de Cap de Gata (Almería), à 36 45' N.

Mot clés: Climat, Pluviométrie, Isohyètes, Espagne péninsulaire et Baléares. 
Desde el último tercio del siglo XIX comienzan a realizarse los primeros mapas pluviométricos relativos a la Península Ibérica. Se daban los primeros pasos en explicar su comportamiento pluviométrico, tanto por parte de autores españoles como de otros países europeos, estos últimos serían los pioneros en intentar aproximarse a una clasificación de las causas de la distribución de la lluvia media de esta Península tan marítima, por estar ampliamente abierta hacia el océano Atlántico y a su vez tan continental, por su singular configuración fisiográfica lo que revertiría en un aislamiento, en relación a las masas aerológicas oceánicas, precisamente aquellas que conllevan los mecanismos pluviométricos fundamentales. De tal manera que conocidos autores, entre ellos podríamos citar a Dantin Cereceda, Hessinger, Masach Alavedra, Birot, González Quijano, han constado o insinuado el hecho de ser la Ibérica de las tres penínsulas mediterráneas la más seca, la que menos se aprovecha de las precipitaciones de origen oceánico.

En 1912 Dantin Cereceda, con un sentido profundamente geográfico, como sólo los muy iniciados en la investigación pueden lograr transmitir, escribía: «ningún fenómeno climatológico tiene mayor influencia sobre la actividad entera de la geografía que el de la lluvia; ella crea y mantiene los ríos, escultores del relieve, dirige la erosión, forma el suelo». Palabras que aún estando vigentes, recobran máxima actualidad hoy día en España sometida a una pertinaz sequía, en un momento de las postrimerías del siglo XX donde todo el mundo escribe sobre cambios climáticos por doquier y creen ver ascensos del nivel marino, incluso por parte de prestigiosos científicos. Se generaliza la idea de que el clima está cambiando, se especula acerca del clima que hay o el clima que viene y todo ello sin un conocimiento profundo sobre el comportamiento climático durante el siglo pasado y el siglo XX. No tenemos la mínima duda de que sobre cambio climático global se puede especular hasta la saciedad, dependiendo de la bibliografía utilizada, denominada «espacializada», «puntera» o «novísima» y de la capacidad de imaginación asistida que pongamos. ¡Y no digamos nada de aquellos organismos internacionales de competencia sobre el clima!, caso de PNUMA, OMM o la Conferencia Mundial sobre el clima, etc., tutelados todos ellos por la ONU, donde las declaraciones alarmistas proliferan amenazantes, a modo de desatinos sonorosos. Pues convergen en ellos múltiples intereses, geopolíticos, medioambientales, estratégicos y económicos.

Como apuntábamos anteriormente las palabras de Dantin recobran singular sentido en España, país en el que la precipitación en todas sus regiones poseen un régimen anárquico en su distribución anual, marcando la pauta climatológica. Ni siquiera Galicia, Asturias o Cantabria escapan a este rasgo específico del régimen pluviométrico ibérico (Martín Vide, 1994).

Hay autores cuya aportación a este tema de investigación ha sido fundamental y hoy son clásicos de cita obligada, por su aportación al conocimiento del clima de España como Hellmann, Angot, González Quijano, Lorenzo Pardo, Semmelhack, Gaussen, Lautensach, F. Huerta, Font Tullot, García de Pedraza o Linés Escardó. Nosotros en este estudio hemos querido enriquecer la cartografía ya existente con el mapa pluviométrico medio de España peninsular y Baleares, para el período internacional 1961-1990. El cual he trazado personalmente en el departamento de Geografía de la Universidad de Murcia.

No debemos perder la perspectiva, aunque esto es bien sabido, que la medida absoluta de la precipitación no es nada fácil de hallar. La altura de agua que registra un pluviómetro (lluvia y precipitación sólida), independientemente del tamaño y de su forma, corresponde parcialmente a la realidad del agua recibida (MURRIEL FERNÁNDEZ y MARTÍN ARANDA, 1973). El agua puede también alcanzar el suelo bajo la forma de condensaciones directas, como el rocío, escarcha, u ocultas, esto es, las que se condensan directamente en el suelo. De cualquier manera, la determinación de la totalidad de agua que la atmósfera 


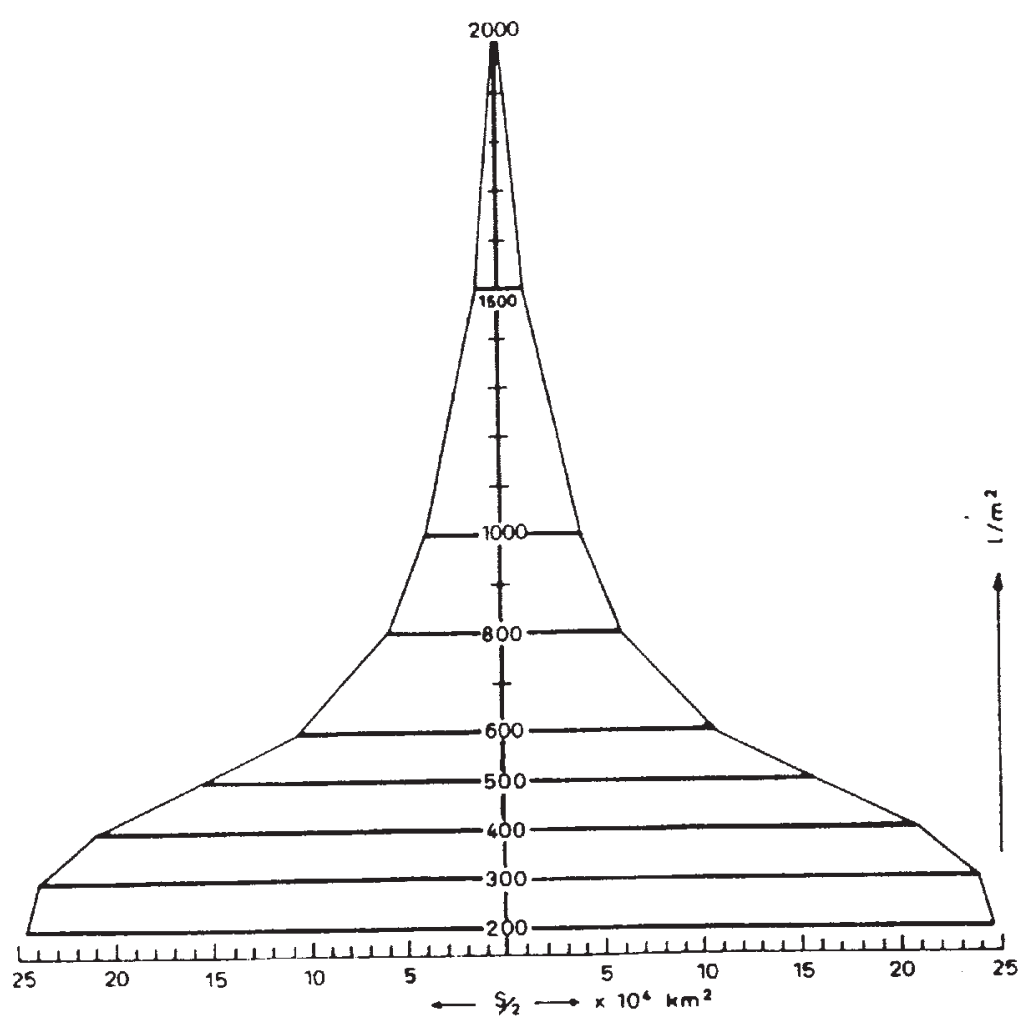

FIGURA 1. Idea esquemática del cálculo del volumen de agua en la España peninsular y Baleares.

cede al suelo en un punto dado, y durante un intervalo de tiempo determinado, no es fácil de determinar con precisión.

Para la realización del mapa pluviométrico, la tarea ha sido complicada: un total de 689 estaciones pluviométricas con datos completos referentes a un período de 30 años, lo cual significa un volumen de algo más de 268.710 números. El mapa ha sido trazado a escala 1:1.000.000, la densidad de la red por cada 1.000 kilómetros cuadrados es de 1,4 para el territorio español, de casi medio millón de $\mathrm{km}^{2}$ de extensión, densidad por otra parte aunque no es la deseable, si es aceptable; únicamente 689 observatorios reunían las series de 30 años completas y con datos enteramente fiables. Existen más de 4.000 estaciones pluviométricas repartidas por el territorio nacional, más con lagunas graves de 10, 15, incluso 20 años, para el período analizado. Nosotros, por nuestra experiencia personal y considerando la latitud subtropical y especial situación longitudinal de la Península Ibérica, hemos preferido intencionadamente no hacer uso del ordenador y programas de correlaciones de base matemático-estadístico, hoy plenamente aceptado por la comunidad científica. Creemos que los valores obtenidos por ese método son teóricos y suelen alejarse bastante de la realidad, por el relieve español tan articulado, con orientaciones, altitudes, sombras orográficas, pasillos orográficos, tan diversos.

Con la finalidad de establecer una relación más profunda entre las isoyetas y la topografía regional, se han dibujado las isoyetas del mapa medio sobre el mapa de la misma escala 
del Instituto Geográfico Nacional, facilitando enormemente la interpretación al lector. Para el trazado de las isoyetas se han tenido en cuenta una serie de factores: los valores de la precipitación, el relieve, y excepcionalmente, el gradiente pluviométrico de altitud; éste último lo hemos utilizado esporádicamente. Lamentablemente, los datos de los pluviómetros totalizadores están frecuentemente falseados en las montañas en razón a la fuerza del viento. De tal manera que las isoyetas trazadas en las Cordilleras Españolas, responden a una selección de los totalizadores, y no aplicando el criterio, excelente por otra parte, del gradiente de altitud, que en realidad no se puede calcular por fórmula alguna, sino que se deduce en función de los datos pluviométricos observados; en el caso de no haber totalizadores, en la montañas importantes se ha adoptado la isoyeta de la estación meteorológica más elevada sobre el nivel del mar.

Un hecho significativo es, por ejemplo, la atribución por parte de H. Lautensach de 2.000 a 2.400 mm., a las cotas más elevadas de Sierra nevada para el período 1931-1960, aplicando el gradiente de altitud, cifra en cualquier modo exagerada. Nosotros hemos comprobado que la vertiente suroccidental de Sierra Nevada, que posee una óptima orientación pluviométrica —en relación a la circulación atlántica de Poniente (NW., W. y SW.) - , apenas alcanza los $1150 \mathrm{~mm}$. en Durcal «Prado Carnero», a 2340 metros de altitud, en un período de 20 años (1956-1978). En cambio en las cotas elevadas del Mulhacén, con una orientación menos favorable, no llegan a los $900 \mathrm{~mm}$., valores mucho más bajos que los que Lautensach atribuía en su investigación a la elevada Serranía Andaluza, aplicándole el criterio del gradiente de altitud. En definitiva, para el trazado de las isoyetas no sólo se deben tener en cuenta los valores de la precipitación, en sí mismos, sino que es necesaria una lógica interpretación de estos datos numéricos. Estamos de acuerdo con H. Lautensach, cuando escribía «el sencillo método de trazar las distancias proporcionales entre puntos fijos, no tiene la menor esperanza de ajustarse a la realidad» (LAUTENSACH, 1971).

\section{El mapa pluviométrico de España}

Los pioneros en el trazado de mapas de isoyetas sobre el solar Ibérico, son climatólogos alemanes y franceses. Hasta los años veinte todos ellos están trazados basándose en un pequeño número de estaciones, a causa de la escasa red de observatorios en el siglo XIX y realizados sobre mapas a pequeña escala.

Los más antiguos son los de Hellmann, G.: Distribución de la lluvia en la Península Ibérica. Revista de Montes, IV, Madrid 1880, pp. 102-110. y Die Regenverhältnisse der Iberischen Halbinsel. Zeitscher. Ges. Erdk., 23, Berlin 1888, pp. 307-400. Karte escala 1: 7.500.000.

Hann, J.: Niederschlagskarte in der neuen Ausgabe von Berghaus Physikal. Atlas. Gotha 1886/87 y el publicado por Angot, A.: Régimen des pluies de la Peninsule Ibérique. Ann. Bur. Cent. Metr. France, 1893. París 1895, pp. 157-194. Escala 1: 1.000.000.

Un nuevo paso lo da fischer, Th.: Die Iberische Habinsel in: A. Kirchhoff, Länderkunde von Europa II, 2. Wien, Prag, Leipzig 1893. S. 655. Diederschlagskarte ist eine Wiedergabe deerr con Hellemann, mit kelinen Weränderungen, die orographie Stärker berücksichkingen.

Tras unos mapas pluviométricos parciales de Semmelkack y Patxot, sobre el Noroeste peninsular y Cataluña, respectivamente, hay que esperar a 1920, cuando se publica por la Dirección General del Instituto Geográfico y Estadístico: Mapa medio de precipitación de España. Escala 1: 3.000.000. Ann. Observatorio Central Meteorológico, Madrid.

Posteriormente González Quijano, P.M. publica: 1) La lluvia en la Península Ibérica durante el quinquenio 1916-1920. Rev. Obras Públicas. Nr. 2428 (1 de mayo de 1925). 
Mapa de isoyetas escala 1: 2.500.000. 2) Avance para una evaluación de la energía hidráulica en España. Ministerio Obras Públicas. Consejo de Energía. Madrid 1932. Mapa de precipitación media 1916-1925.

En 1932 publica Semmelhack, W.: Nierderschlagskarte der Iberischen Halbinsel. Periode 1861-1900. Ann. d. Hydr. usw. 60, 1932, s. 28-32 u. Taf. 7. y el mismo año Lorenzo Pardo, L.: Plan general de Obras públicas. Servicio Central Hidráulico, Madrid, incluye un mapa pluviométrico en el tomo I. Mapa de isoyetas escala 1: 4.000.000.

Respecto a Portugal destaca los mapas pluviométricos de Amorin Girao, A. de.: 1) Atlas de Portugal. Coimbra. 1941. Taf. V. Pluviometría. Mapa escala 1: 1.500 .000 y 2) Geografía de Portugal. Porto 1941. Distribuiçao da chuva. Escala 1: 2.500.000.

En 1942 publica el Ministerio de Obras Públicas: Mapa pluviométrico de España y Portugal. Madrid. Y en ese mismo año edita el Servicio Meteorológico Nacional: Mapa pluviométrico de España. Período 1913-1932. Escala 1: 1.500.000. Madrid. Un año después, en 1943, en Portugal publica Amorin Ferreira, H.: Distribuiçao da chuva no territorio do Continente português. Escala 1: 1.000.000. Lisboa.

En 1946, publicado por el Consejo Superior de Investigaciones Científicas, aparece la obra de González Quijano, P.M.: Mapa pluviométrico de España. Madrid, con un mapa en nueve hojas, Escala 1: 800.000. Y en 1949 aparece el mapa pluviométrico de Gaussen, H.: La carte de pluviosité de l'Espagne. Mélanges geographiques en hommage à Daniel Faucher. Toulouse 1948. Escala 1: 3.600.000.

Dos años después, en 1951, publica Lautensach, H.: Die Nierdersclagshöhen auf der Ibersichen Halbinsel. Eine Geographische Studie. «Petermanns Geog. Mitteilungen» 1951. 3Quart, pp. 145-160. Mapa de isoyetas Escala 1: 1.500.000 y editado en castellano, posteriormente en 1971, por el Servicio Meteorológico Nacional.

En marzo de 1969, publica Huerta, F.: La lluvia media de la España peninsular en el período 1931-1960. S.M.N., Madrid. Con un mapa de precipitación media Escala 1: 1.000 .000 .

En 1977, publica Suzanne Daveau: Répartition et Rytme des precipitations au Portugal. Centro de Estudios Geográficos, Lisboa. Con un mapa pluviométrico medio de Portugal; período 1931-1960 y a escala 1: 250.000.

Y finalmente, en 1983, el I.N.M., edita un mapa pluviométrico de España a Escala 1: 3.000 .000 .

\section{Factores de las precipitaciones en España}

Los mecanismos pluviométricos en España puede entenderse en función de su ubicación marginal (posición de abrigo) sobre el flanco meridional del flujo zonal del Oeste (Westerlies). Por consiguiente, las fases de onda planetaria de la corriente zonal del Oeste van a explicar la alternancia de períodos húmedos y secos, en función de que estemos en la zona de convergencia (vaguada o valle planetario) o zona de divergencia (dorsal o cresta planetaria). Estas distintas fases de la corriente en chorro se ejercen con frecuencia en la época más fría del año (octubre-mayo). Fundamentalmente la originalidad del clima de España aparece bien definida en la estructura de su dinámica atmosférica - tipos de tiempo- y en la circulación a la topografía de $500 \mathrm{mb}$. Para entender la climatología ibérica es indispensable analizar las capas altas de la atmósfera, ya que en superficie numerosas contradicciones se acumulan y no nos permiten realmente descubrir sus constantes. E incluso los sistemas nubosos representados en superficie, herencia (fundada en consideraciones sinópticas) de la frontología noruega «Escuela de Bergen, 1922», no ofrecen siempre del todo relaciones paralelas con las precipitaciones, puesto que a veces se 
desencadenan lluvias generales e intensas con la ausencia de verdaderos sistemas frontales e incluso otras veces el paso de frentes muy marcados en superficie apenas se muestran activos y escasean las lluvias, o bien desencadenan lluvias generales.

Por tanto, el flujo zonal a $500 \mathrm{mb}$., es condicionante del tiempo atmosférico en nuestro espacio sinóptico y tiránico en cuanto a los mecanismos pluviométricos. En nuestras latitudes, las ondas planetarias de la circulación zonal dirigen el comportamiento de las perturbaciones de superficie. Mientras que en el norte y oeste de Europa se constata que la frontología superficial posee una cierta independencia frente al flujo superior.

El análisis dinámico nos pondrá de manifiesto que el límite geofísico septentrional del clima subtropical mediterráneo aparece muy claro en el flanco norte de la Península Ibérica, Mediterráneo Occidental y Oriental; se trata en definitiva de un límite esencialmente aerológico. En cambio, sobre las regiones templadas y templado-frías de la Europa del Oeste, Península Escandinava y llanura rusa, las condiciones de la circulación en las capas altas son homogéneas - grosso modo- y las diferencias climáticas son debidas preferentemente a los efectos de su ubicación geográfica (proximidad o alejamiento del mar) o a los efectos del relieve. Aquí, las condiciones de altitud no son tan determinantes, mientras que en los archipiélagos ibéricos, Península Ibérica, Norte de África, cuenca mediterránea, Mar Negro y Mar Caspio el relieve y la situación, en relación a las corrientes aerológicas, no explican su singular comportamiento; hay que ascender necesariamente a las capas altas. Las condiciones termodinámicas de niveles altos son más determinantes y tiranizan a las condiciones de superficie, cuyo diagnóstico es más secundario. Las isotermas y la curvatura del flujo en altura (ciclónico o anticiclónico) darán lugar a la inestabilidad vertical dinámica para la formalización de los mecanismos desencadenadores.

El espacio físico está acotado aproximadamente entre $36^{\circ}$ y $44^{\circ} \mathrm{N}$, constituyendo una zona de transición que pone en comunicación ámbitos geográficos distintos pero que se complementan: influencia del Mediterráneo por su flanco oriental (vertiente levantina) y del Atlántico (vertiente occidental) por otra. La península presenta un relieve en conjunto fuertemente compartimentado, debido a que alternan alineaciones montañosas, depresiones, llanuras y valles que actúan a modo de pasillos naturales y que conectan el interior continental con la periferia.

La topografía impone unas condiciones de canalización, de corredores orográficos, barlovento/sotavento, a las masas de aire alógenas que nos visitan, con sus características propias (temperatura, humedad, nubosidad, detención, descenso) quedando en parte modificadas. Así la disposición orográfica de la Península, favorece, a grandes rasgos, la extensión de las influencias marítimas atlánticas: los altos relieves terciarios -Pirineos, Cordillera Cantábrica, Sistema Central, Montes de Toledo, Sierra Morena y Cordilleras Béticas (en menor grado) - presentan una disposición preferentemente zonal (oeste-este), de tal manera que no se oponen al flujo zonal marítimo de Poniente, dominante en la latitud de Europa. La excepción lo constituye el Sistema Ibérico, que corre transversalmente de NW a SE, y que delimita dos ámbitos diferentes: de influencia atlántica, de una parte, a barlovento del mismo y, de otra, mediterránea a sotavento. Igualmente el flanco oriental de las Cordilleras Béticas, a sotavento de las influencias atlánticas, presentan un claro dominio del ámbito mediterráneo en sus caracteres termopluviométricos. La trascendencia biogeográfica de estas alineaciones topográficas (Sistema Ibérico y Cordilleras Béticas), en su sector oriental, se deja sentir negativamente con respecto a los flujos húmedos superficiales procedentes del océano, sometiéndolos a un intenso efecto foehn. Por el contrario, en relación con los vientos de levante originarios del Mediterráneo, actúa en este flanco oriental con ascensionalidad, detención y precipitaciones.

Tal configuración orográfica determinará dos características: 
1) Que la influencia atlántica se introduce hasta alcanzar los flancos orientales de ambas submesetas, aunque degradándose según se avanza de oeste a este.

2) Tal disposición no permite el libre paso de masas de aire meridianas del $\mathrm{N}$ y NE.

Centros de acción, masas de aire y discontinuidades frontales, constituyen los pilares fundamentales de la dinámica atmosférica regional. El anticiclón de Azores, los anticiclones polares oceánicos, los anticiclones continentales europeos, el anticiclón peninsular, la depresión de Islandia, la depresión Gibraltareña, la depresión del golfo de Génova, la depresión térmica peninsular, la depresión térmica Sahariana, constituyen los centros de acción atmosféricos que con su posición regulan la dinámica del clima ibérico a lo largo del año. La situación de la Península Ibérica no está en la trayectoria normal de las perturbaciones del frente polar; la mayor parte de las perturbaciones atmosféricas de la Península, región del estrecho y Mediterráneo Occidental se caracteriza por ser, en su mayoría, nuevas formaciones o bien reactivizaciones de otras existentes que pueden considerarse como nuevas.

No cabe duda que los caracteres del tiempo y especialmente los mecanismos de la precipitación de la Península Ibérica se desarrollan paralelamente y se subordinan, esto es, son dependientes, en relación a las condiciones de abrigo. El concepto de «abrigo» se entiende en una doble vertiente: aerológica y topográfica. De todos es conocido el abrigo topográfico puesto de manifiesto por geógrafos. Los rasgos continentales de Alberta, Montana, Woyomin, resultan en realidad, aparte de su lejanía a las costas, del estancamiento de masas de aire oceánicas al oeste de la cordillera de las Rocosas. Y así, mientras en la fachada pacífica de la antigua Columbia Británica, Vancuver y el piedemonte occidental registran precipitaciones anuales superiores a 1.500/2.000 mm., a sotavento de la gran cordillera, en Alberta o Nevada, los grandes bosques son sustituidos por la pradera o estepa, con vientos frecuentes de tipo foehn, recalentados adiabáticamente muy comúnmente en toda estación (cuando la circulación es zonal) y lluvias anuales inferiores a los $400 \mathrm{~mm}$.

Pero paralelamente a ello, los tipos de tiempo y las precipitaciones en la Península se desarrollan prioritariamente en relación con el abrigo aerológico; esto explica el carácter del tiempo perturbado o en calma, en definitiva, dicho en términos más específicos, según que estemos bajo curvatura - ciclónica o anticiclónica- del flujo zonal al nivel de la topografía absoluta de $500 \mathrm{mb}$.

La latitud de la Península Ibérica es un hecho inamovible y prioritario a tener en cuenta. La proximidad y presencia en niveles altos del anticiclón de las Azores, va a provocar la sequía en largos períodos, aridez tanto más acusada cuanto más hacia el Sur y más hacia el Este nos dirijamos a través del solar ibérico; esto es, mayor proximidad a la alta subtropical, por un lado (abrigo aerólogo) y, de otro, posición de abrigo topográfico. España, como el Mediterráneo y el Norte de África, están en efecto situados casi siempre en el borde sur del torbellino circumpolar, en el flanco meridional por donde discurre el Jet Polar y las perturbaciones ligadas a la ondulación del frente polar en superficie. España por su ubicación subtropical escapa de la turbulencia de la zona polar de mezcla situada, por lo general, al norte del paralelo $40^{\circ}$, espacio en donde las irrupciones frías desencadenan mecanismos ciclogenéticos intensos. Interesa destacar, pues, esta posición de abrigo aerológico, en relación a la circulación polar, quedando nuestra región en el flanco sureste del mínimo de Islandia, ligado al anillo planetario de bajas presiones Subpolares; anillo que alcanza una extensión superficial menor que en el hemisferio Austral y, por tanto, esto es un hecho condicionante. Por nuestra latitud, los caracteres del tiempo dependen estrechamente de los mecanismos polares y no tropicales, especialmente, en relación a la producción de preci- 
pitaciones (FONT TULLOT, 1983). Las irrupciones provienen del sector Norte (N y NE) y sector Oeste (NW, SW y W) o incluso sector Sur. Las corrientes perturbadoras de origen tropical no intervienen de manera eficaz, pues incluso las perturbaciones del Oeste/ Suroeste/Oeste, se trata en definitiva de aire polar que ha descrito un gran arco de herradura en el Atlántico Norte (aire polar marítimo de retorno), nunca aire tropical marítimo, que en todo caso, nos alcanza con tiempo bueno pues se trata de masas de aire cálido que al dirigirse a territorios de latitud mayor, más fríos, se produce un continuo enfriamiento en su base, implicando un aumento de la estabilidad atmosférica. Los mecanismos pluviométricos se desencadenan cuando transgresiones de aire polar penetran en altitud sobre nuestra vertical, con curvatura ciclónica y con una temperatura lo suficientemente baja como para que la inestabilidad dinámica y termodinámica se desarrolle.

El clima de España puede entenderse, con cierta nitidez y a grandes rasgos, en función de su posición marginal (posición de abrigo) sobre el flanco meridional del flujo zonal del Oeste (Westerlies). Por consiguiente, las fases de la onda planetaria de la corriente zonal del Oeste van a explicar la alternancia de períodos húmedos y secos, según que estemos en la zona de convergencia (vaguada o valle planetario) o zona de divergencia (dorsal o cresta planetaria). Estos estados diferentes de las distintas fases de la corriente en chorro se ejercen frecuentemente en la estación invernal, primavera y otoño y muy raramente en verano. Los ejes de las dorsales planetarias a $500 \mathrm{mb}$. y los flancos orientales de las dorsales son áreas de divergencia en superficie, el aire al descender se recalienta adiabáticamente y se estabiliza; por el contrario, el eje de las vaguadas planetarias y los flancos orientales de las vaguadas son áreas de convergencia dinámica y, por tanto, animados de movimientos ascensionales ciclónicos del aire.

El flujo zonal a $500 \mathrm{mb}$. es, si no determinante, sí al menos condicionante máximo del tiempo en nuestro espacio sinóptico, y tiránico en cuanto a los mecanismos pluviométricos. En nuestras latitudes las ondas planetarias de la circulación zonal superior dirigen obligadamente el comportamiento de las perturbaciones de superficie. Mientras que en el Norte y Oeste de Europa se constata que la frontología superficial posee una cierta independencia frente al flujo superior.

\section{La lluvia media de la España peninsular y Baleares en el período 1961-1990}

No cabe duda que la distribución de las isoyetas en España, con sus diferentes núcleos de máxima o mínima precipitación ofrecen un aspecto complejo. Sin embargo, hay un gran paralelismo con una representación cartográfica de su topografía. Los núcleos orográficos se corresponden a los núcleos pluviométricos más importantes; la precipitación aumenta con la altitud, aunque no necesariamente, siempre intervienen factores como la orientación, exposición a las corrientes aerológicas oceánicas, ubicación geográfica (periferia/interior), (Mediterráneo/Atlántico), entre otros. Los mínimos pluviométricos se desplazan a aquellos enclaves más deprimidos topográficamente (cuencas de los ríos, hoyas, depresiones interiores), llanuras litorales o enclaves a sotavento de altos relieves orográficos. De tal forma que en la descripción geográfica del mapa pluviométrico de España, vamos a denominar a los entes pluviométricos a través de los nombres geográficos de los sistemas correspondientes.

La pluviometría española presenta cifras muy desiguales, en relación a lo compartimentado de su relieve. Así entre el N/NW y el SE peninsular se producen los contrastes más acusados entre los que se origina el máximo gradiente vegetal y pluviométrico. Degradándose la cobertura vegetal arbórea de Norte a Sur, igualmente las precipitaciones en ese mismo sentido van decreciendo. A lo largo del meridiano $2^{\circ} \mathrm{W}$, se origina el 
máximo gradiente, desde el observatorio de Articutza (Navarra Atlántica), a $43^{\circ} 12^{\prime} \mathrm{N}$ y con $2.661 \mathrm{~mm}$., hasta los $156 \mathrm{~mm}$. de Cabo de Gata «Faro» (Almería) a 36 45' N.

La distinción entre España húmeda y España seca, valiéndonos del límite de la isoyeta anual de $800 \mathrm{~mm}$., sería una primera aproximación a la síntesis pluviométrica española. Introduciendo un nuevo matiz, podríamos diferenciar tres territorios pluviométricos:

I. España Húmeda, definida por la isoyeta de $800 \mathrm{~mm}$., con precipitaciones iguales o superiores a este valor.

II. España de Transición, enmarcada entre las isoyetas de 800 y $300 \mathrm{~mm}$.

III. España Árida, definida por la isoyeta de $300 \mathrm{~mm}$., con precipitaciones inferiores a este valor.

En una reproducción del mapa del Instituto Geográfico Nacional, escala 1:1.000.000, se han medido con un planímetro las superficies representadas por cada uno de esos gradientes pluviométricos, resultando que: La España húmeda representa $95.280 \mathrm{~km}^{2}$, o sea, el 19,1\% del territorio. La España de transición ocupa $390.226 \mathrm{~km}^{2}$, con el 78,4 \% del espacio y la España árida, representa $12.210 \mathrm{~km}^{2}$, o sea, el $2,4 \%$ del territorio. La propuesta de clasificación es un intento de simplificar y clarificar la descripción geográfica del mapa pluviométrico.

\section{A. La España Húmeda (territorio con precipitaciones superiores a $800 \mathrm{~mm}$.)}

\section{Núcleo Galaico-Duriense y cordilleras Pirenáica y Cantábrica}

Es el área pluviométrica más importante de España, incluye las cuatro provincias gallegas, Asturias, Santander, País Vasco, Navarra Atlántica y parte de las provincias de Gerona, Lérida, Huesca, Zaragoza, Burgos, Palencia, León y Zamora. La isoyeta de 800 $\mathrm{mm}$. parte desde la frontera portuguesa, introduciéndose en la provincia de Zamora por el embalse de Valparaíso, se encurva hacia el Norte, atravesando al sur del embalse de Villameca al oeste de León y continua su trazado hacia el NE, para seguir luego más o menos regularmente de Oeste a Este en el piedemonte meridional de la cordillera Cantábrica. Pasa por Sopeña de Curueño (León), embalse de Requejada, Condado de Treviño, Estella, Pamplona, siguiendo hacia el Este, bordeando los Pirineos, embalse de Yesa, pasa al Sur de Berdún, Jaca y Sabiñánigo, atraviesa el embalse de Mediano sobre el río Cinca, La Seo de Urgel, Ripoll, Olot y Montes Alberes.

Dentro de este núcleo la isoyeta de $1.000 \mathrm{~mm}$. llega a todo el litoral cantábrico y gallego, a excepción de un pequeño enclave en el litoral asturiano, entre las proximidades cabo de Peñas y Gijón. La isoyeta de 1.000 mm. por el Sur parte de Portugal, introduciéndose en la provincia de Zamora por Puebla de Sanabria, sigue hacia el Norte, al Este del lago de Sanabria y engloba la sierra de la Cabrera, dirigiéndose hacia el Oeste, a través de la cuenca del río Sil, pasando al Sur de la sierra de Ancares se dirige al Este, a través del embalse de Luna, embalse de Porma, Reinosa, continua al Norte de las Ciudades de Vitoria y Pamplona, bordea el piedemonte pirenaico y termina en el Pirineo oriental gerundense, englobando La Molina y Nuria. Dentro de la isoyeta de $1.000 \mathrm{~mm}$., aparecen cinco núcleos de $1.500 \mathrm{~mm}$.: el más extenso es el gallego, afectando a gran parte de las provincias de Coruña y Pontevedra, con un máximo de $1.952 \mathrm{~mm}$. en el aeropuerto de Vigo «Peinador».

El segundo núcleo de $1.500 \mathrm{~mm}$. aparece enclavado en la provincia de Zamora - comarca de Sanabria - sierra de la Cabrera, principalmente en su vertiente suroccidental, con 1.625 y 1.604 mm., en El Fraile y Presa Cárdena, respectivamente. 
El tercer núcleo de $1.500 \mathrm{~mm}$., se ubica en el macizo de los Picos de Europa, con un máximo de $1.817 \mathrm{~mm}$. en Amieva «Restaño».

El cuarto núcleo de $1.500 \mathrm{~mm}$. se emplaza en Cantabria, en la cuenca alta del río Pas, con $1.773 \mathrm{~mm}$. en Villacarriedo.

El quinto núcleo parte de la costa al Este de San Sebastián, continua hacia el oeste, abarcando la mayor parte de la provincia de Guipúzcoa, Navarra Atlántica, continua hacia el Oeste, atraviesa Canfranc y vira al Norte, a lo largo de la cuenca del río Gállego, hasta la frontera con Francia. Dentro de él, hay un núcleo de más de $2.000 \mathrm{~mm}$. en el valle de Baztán, alcanzándose $2.661 \mathrm{~mm}$. en Articutza, que constituye el máximo pluviométrico de España.

\section{Núcleos del Sistema Central}

Desde Portugal se introduce la isoyeta de $800 \mathrm{~mm}$., que incluye Sierra de Gata, Las Hurdes, Peña de Francia, sierra de Candelario, Sierra de Gredos, sierra de la Paramera, sierra de Guadarrama y Somosierra, circunvalando toda la cordillera Central Divisoria, izándose en uno de los núcleos hidrográficos más importantes de España. Destaca 1.876 $\mathrm{mm}$. en el observatorio de Guisando El Risquillo y $1.361 \mathrm{~mm}$. en Serranillos, en la sierra de Gredos; y 1.409 mm. en el Puerto de Navacerrada, en la sierra de Guadarrama.

\section{Núcleo de los Montes de Toledo}

Se reduce exclusivamente al pequeño enclave de la sierra de Guadalupe y estribaciones próximas, con $803 \mathrm{~mm}$. en Alía.

\section{Núcleo de Sierra Morena}

Se extiende, en el sector más occidental del sistema montañoso, ubicado sobre sierra de Aracena y estribaciones, destacando $1.142 \mathrm{~mm}$. en Cortegana y $1.108 \mathrm{~mm}$. en Jabugo.

\section{Núcleos de los Sistemas Béticos}

El núcleo más relevante lo constituye el configurado en el extremo meridional de la Península; comprende la mayor parte de la provincia de Cádiz y sector occidental de Málaga. La isoyeta de $800 \mathrm{~mm}$., arrancando de Algeciras, bordea la sierra de Carbonera, sierra Bermeja, serranía de Ronda y sierra de Ubrique, destacando $1.117 \mathrm{~mm}$. en Gaucin y el máximo se centra en torno a la sierra de Ubrique, donde el observatorio de Grazalema «Benamahoma» anota $1.168 \mathrm{~mm}$.

Un segundo núcleo muy húmedo aparece enclavado en la sierra de Cazorla, en el alto Guadalquivir, con $1.137 \mathrm{~mm}$. en Cazorla «Vadillo Castri», extendiéndose a las sierras del Pozo, Segura, Calar del Mundo y Alcaraz. Por otro lado, un tercer núcleo se sitúa en el curso alto del río Guadalbullón y sierra Mágina, con 871 mm. en Valdepeñas de Jaén.

\section{Núcleo Catalán}

Asociado al macizo montañoso de Montseny, con $1.073 \mathrm{~mm}$. en Turo del Home. 


\section{B. La España Árida. (Territorio con precipitaciones inferiores a $300 \mathrm{~mm}$.)}

Abarca aproximadamente el 3,4 \% del territorio español, localizado preferentemente en el SE peninsular. Gran parte de las provincias de Almería y Murcia quedan englobadas en este dominio y enclaves de las altiplanicies de Granada, Albacete y sur de Alicante. Hay un mínimo secundario que rebasa ligeramente los $300 \mathrm{~mm}$. en el bajo Duero, al NE de la Armuña con $311 \mathrm{~mm}$., en el observatorio de Alaejos (Valladolid).

I. La Estepa litoral del SE español. El área con precipitaciones inferiores a $300 \mathrm{~mm}$. cubre amplios espacios de las provincias de Alicante, Murcia y Almería, correspondiéndose con la estepa litoral. La isoyeta de $300 \mathrm{~mm}$. parte de la costa andaluza almeriense, al oeste de Adra, bordea el macizo de Sierra de Gádor, se introduce por el curso alto del rio Andarax virando al Este, continuando por el piedemonte de sierra de los Filabres, continua por Lubrin, penetra profundamente por la cuenca del río Almanzora, continuando por el flanco sur de sierra de las Estancias, dirigiéndose con posterioridad hacia el Norte, englobando $\mathrm{D}^{\mathrm{a}}$ Inés, dejando fuera el macizo húmedo de sierra de Espuña. Continua hacia el Norte por el curso alto del río Segura, interesando parte del altiplano murciano, pasa por Jumilla y por el sur de sierra de la Pila, continuando de oeste a este, por la cuenca baja del río Vinalopó, introduciéndose en el Mediterráneo al sur de Alicante. Dentro de este área, aparecen dos núcleos muy áridos. Uno ubicado al sur de la provincia de Alicante, destacando $238 \mathrm{~mm}$. en Elche y $236 \mathrm{~mm}$. en Laguna de La Mata y otro, emplazado más hacia el Sur, sobre la sierra de Cabo de Gata, donde el observatorio de Cabo de Gata «Faro» registra 156 mm., que constituye el mínimo pluviométrico de España y del continente europeo (cifras comparables aunque ligeramente superiores se registran en el SE de Rusia, costa noroccidental del Mar Caspio; en la costa oriental asiática del Caspio, los valores son incluso inferiores).

\section{La España de Transición. (territorio con precipitaciones comprendidas entre 800 y $300 \mathrm{~mm}$.)}

Abarca la mayor parte del suelo español $(78,4 \%)$. Introduciéndose por las cuencas de los grandes ríos atlánticos (a excepción del Miño) y cuencas de los ríos levantinos, catalanes y cuenca del río Ebro. Abarca pues toda la cuenca del Guadalquivir, País Catalán, País Valenciano y ambas submesetas.

\section{Análisis cuantitativo del mapa pluviométrico}

Hemos llevado a cabo una valoración, grosso modo, del volumen total de agua que representan las isoyetas dibujadas sobre el mapa. Para tal fin, en un mapa escala 1: 1.000.000, hemos medido con un planímetro las áreas comprendidas entre los diferentes intervalos pluviométricos utilizados; 2.500-1.500, 1.500-1.000, 1.000-800, 800-600, 600-500, 500$400,400-300,300-200$ y 200-100 mm. Las áreas que resultaron se consignan en el cuadro adjunto. El volumen de agua precipitada se calcula como volúmenes de tronco de cono limitados por las dos superficies sucesivas de áreas s y s' y altura h por la fórmula.

$$
\mathrm{V}=1 / 3\left(\mathrm{~s}-\mathrm{s}^{\prime}-\sqrt{\mathrm{s} \cdot \mathrm{s}^{\prime}}\right) \cdot \mathrm{h} .
$$

cuyos resultados son los siguientes: 
Cuadro I

CÁLCULO DEL VOLUMEN DE AGUA

\begin{tabular}{|ccc|}
\hline $\begin{array}{c}\text { Precipitación en } \\
(\mathbf{m m} .)\end{array}$ & $\begin{array}{c}\text { Área: } \mathbf{K m}^{\mathbf{2}} \\
\text { (superficie acumulada) }\end{array}$ & $\begin{array}{c}\text { Volumen } \\
\mathbf{1 0}^{\mathbf{8}} \mathbf{~ m}^{\mathbf{3}}\end{array}$ \\
\hline 1500 & 12.240 & 40,9 \\
1000 & 63.670 & 173,0 \\
800 & 95.280 & 157,8 \\
600 & 169.820 & 261,5 \\
500 & 274.110 & 219,8 \\
400 & 437.146 & 352,4 \\
300 & 485.506 & 461,1 \\
200 & 497.036 & 491,2 \\
100 & 497.716 & 497,3 \\
0 & & 497,7 \\
\hline & & $3.152,1$ \\
\hline
\end{tabular}

En la figura adjunta se ofrece una representación esquemática del cálculo, como ordenadas se toma las precipitaciones y como abcisas las áreas. Para aproximarnos aún más a la distribución de las precipitaciones, se calcularon los volúmenes correspondientes a cada uno de los intervalos de las isoyetas, partiendo de los datos incluidos en el cuadro 1. Las fórmulas aplicada, (Huerta, 1969), se deducen de la figura adjunta.

Los resultados de los cálculos se dan en el cuadro siguiente.

Cuadro II

RESUMEN DEL MAPA PLUVIOMÉTRICO

\begin{tabular}{|rrrrr|}
\hline $\begin{array}{c}\text { Intervalo } \\
\text { de isoyetas }\end{array}$ & $\begin{array}{c}\text { Área } \\
\text { (en Km }{ }^{2} \text { ) }\end{array}$ & $\begin{array}{c}\text { \% } \\
\text { del total } \\
\text { (isoyeta media) }\end{array}$ & $\begin{array}{c}\text { Volumen en } \\
\mathbf{1 0}^{\mathbf{8}} \mathbf{~ m}^{\mathbf{3}}\end{array}$ & $\begin{array}{c}\text { \% } \\
\text { Total }\end{array}$ \\
\hline $2500 / 1500$ & 12.240 & 2,4 & 244,89 & 7,6 \\
$1500 / 1000$ & 51.430 & 10,3 & 642,87 & 20,1 \\
$1000 / 800$ & 31.610 & 6,3 & 284,49 & 8,8 \\
$800 / 600$ & 74.540 & 14,9 & 521,78 & 16,3 \\
$600 / 500$ & 104.290 & 20,9 & 573,59 & 17,9 \\
$500 / 400$ & 163.036 & 32,8 & 733,66 & 22,9 \\
$400 / 300$ & 48.360 & 9,7 & 169,26 & 5,2 \\
$300 / 200$ & 11.530 & 2,4 & 28,82 & 0,9 \\
$200 / 100$ & 680 & 0,2 & 1,02 & 0,03 \\
& 497.716 & & $3.200,30$ & \\
\hline
\end{tabular}

El volumen total medio de agua precipitada sobre España peninsular es de 3.200, $3 \times 10^{8} \mathrm{~m}^{3}$, lo que da como precipitación media para todo el territorio de $642 \mathrm{~mm}$.

En la España húmeda con una superficie de $95.280 \mathrm{~km}^{2}$, el 19,1\% del total, se recoge 
un volumen de $1.172 \times 10^{8} \mathrm{~m}^{3}$ que es el $36,6 \%$ del total y una precipitación media anual de $1.230 \mathrm{~mm}$.

En la España de Transición con una superficie de $390.226 \mathrm{~km}^{2}$, el 78,4 \% del total, recoge un volumen de $1998 \times 10^{8} \mathrm{~m}^{3}$ que es el $62,4 \%$ del total y una precipitación media de $512 \mathrm{~mm}$.

En la España árida, con una superficie de $12.210 \mathrm{~km}^{2}$, el 2,4\% del total, lo que representa un volumen de $29,8 \times 10^{8} \mathrm{~m}^{3}$ que significa el $0,9 \%$ del total y una precipitación media de $237,5 \mathrm{~mm}$.

\section{Conclusiones}

Indicábamos con anterioridad que la precipitación media anual de España peninsular y Baleares, en promedio, era de $642 \mathrm{~mm}$. En cualquier caso, es siempre un valor indicativo, no necesariamente representativo de la precipitación, pues en definitiva es el valor medio entre precipitaciones elevadas de la España húmeda y las extremadamente bajas de la España árida. El valor de la precipitación media anual es concordante con el valor de $709 \mathrm{~mm}$. que nos ofrece (González Quijano, 1946) en su clásica obra, que constituye, además, un auténtico tratado de Climatología. Igualmente ese valor de la precipitación es relativamente concordante con el ofertado por F. Huerta $(687 \mathrm{~mm}$.) para el período internacional 1931-1960. Muestra también analogías con el valor medio de $702 \mathrm{~mm}$. que aparece en el Calendario Meteorofenológico, para el período 1947-1967.

La distribución de las precipitaciones en España, grosso modo, se ajustan a las 6 reglas generales postuladas por Herman Lautensach.

1. Prevalece un descenso pluviométrico y del número de días de precipitación, desde su extremo septentrional a su flanco suroriental (Norte a Sur). Este decremento, en el contexto de las latitudes templadas de las costas occidentales de los continentes, responde a leyes planetarias.

2. Las precipitaciones anuales son superiores en el flanco occidental atlántico que en el oriental mediterráneo. Así se aprecian: Cortegana (Huelva) $1.142 \mathrm{~mm}$. y $238 \mathrm{~mm}$. en Laguna de Torrevieja (Alicante), en las cercanías del paralelo $38^{\circ} \mathrm{N} ; 1952 \mathrm{~mm}$. en Vigo «Aeropuerto» y $692 \mathrm{~mm}$. en el embalse de Oliana (Lérida).

3. Las precipitaciones aumentan con la altitud. Las isoyetas del mapa pluviométrico son reflejo, a grandes rasgos, del de isohipsas. Esto es cierto de una manera muy singular en el Sistema Central.

4. Las precipitaciones disminuyen desde el litoral hacia el interior del solar ibérico, y ello directamente relacionado con la continentalidad y masividad que muestra la Meseta Castellana.

5. A sotavento de las corrientes aerológicas oceánicas que provocan los mecanismos pluviométricos más importantes, la precipitación es escasa; sobre todo en áreas deprimidas topográficamente (hoyas, depresiones,...). La llegada a estos enclaves tanto de masas de aire como de superficies frontales, van asociadas a movimientos de subsidencia atmosférica (La Armuña, Bajo Ebro, desierto de Calanda, desierto de Tabernas, hoya de GuadixBaza, depresión de Abanilla-Fortuna, etc.).

6. Las tierras bajas costeras, islas sin relieve y cabos, son indigentes en lluvias, a excepción del litoral cantábrico y costa gallega, muy perturbados por el continuo trasiego de perturbaciones frontales; mientras que en el resto del territorio, la ausencia de precipitaciones de componente orográfica, es manifiesto. 


\section{Bibliografía}

ALT, E. (1932): «Klimakunde von Mittel und Südeuropa». Del Köppen-Geiger, Handbuch der Klimatologie, Tomo III M., Berlin.

ANGOT, A. (1895): «Régime des pluies de la Peninsule Ibérique». Ann Bur. Cent. Meter. France. Mapa medio de precipitación escala 1:10.000.000. Paris, pp. 157-194.

ARNAUD, G. (1925): «La règion las plus seche d'Espagne». Annales de Géographie, $\mathrm{n}^{\circ}$ 191, XXXIV, París, pp. 470-471.

BIEL LUCEA, A. (1963): «La lluvia en España». Boletín Mensual Climatológico, S.M.N., marzo. Madrid, pp. 2-7.

CAPEL MOLINA, J. J. (1981): Los climas de España. Ed. Oikos-Tau, Barcelona, 435 pp.

DANTIN CERECEDA, J. (1912): Resumen Fisiográfico de la Península Ibérica. Instituto Nacional de Ciencias Físico-Naturales. Trabajos del Museo de Ciencias Naturales, no 9. Madrid.

DIRECCIÓN GENERAL INSTITUTO GEOGRÁFICO Y ESTADÍSTICO (1920): Mapa pluviométrico de España. Escala 1: 3.000.000. Anales del Observatorio Central Meteorológico, 3, Madrid.

DOPORTO, M. (1927): «Las lluvias orográficas. Aplicación a la Sierra de Grazalema». Sociedad Española de Meteorología. Anales, 1. Madrid, pp. 5-12.

ELIAS, F. (1963): Precipitaciones máximas en España. Régimen de intensidades y frecuencias. Dirección General de Agricultura. Madrid, 267 pp.

F. I. R. (1946): «El régimen anual de lluvias en la Península Ibérica». Calendario Meteorofenológico, S.M.N., Madrid, pp. 121-130.

GARCÍA DE PEDRAZA, L. (1963): «La nieve». Boletín Mensual Climatológico, S.M.N., diciembre, Madrid, pp. 2-7.

GAUSSEN, H. (1952): «La pluviometrie Ibérique», Rev. des Pyrénées et du Sud-Ouest. XXIII (3). Toulouse, pp.153-162.

GAUSSEN, H. (1948): «La carte de pluviosité de l'Espagne». In Melanges Geographiques offerts en hommage à Daniel Faucher, Mapa de isoyetas, escala 1:3.600.000, Toulouse, pp. 352-358.

GONZÁLEZ QUIJANO, P. M. (1925): La lluvia en la Península Ibérica durante el quinquenio de 1916-1920. Rev. de Obras Públicas, 2428. Con mapas de isoyetas, Escala 1:2.500.000, Madrid.

GONZÁLEZ QUIJANO, P. M. (1932): Avance para una evaluación de la energía hidráulica en España. Min. Obras Públicas. Consejo de Energía. Mapa de precipitación media 1916-25. Madrid.

GONZÁLEZ QUIJANO, P. M. (1946): Mapa pluviométrico de la Península Ibérica e islas Baleares. Cons. Sup. Inv. Cient. Mapa escala 1: 800.000 en 9 hojas. Madrid. 574 pp.

HELLMAN, G. (1888): Die Regenverhältnisse der Iberische Halbinsel Zeits. Ges. Erdk. Un mapa en colores escala 1:7.500.000, Berlín 23.

HELlMAN, G. (1880): «Distribución de las lluvias en la Península Ibérica». Rev. de Montes, IV, Madrid, pp. 102-110.

HERNÁNDEZ PACHECO, P. (1941): «La intensidad de las precipitaciones atmosféricas y la acción erosiva de las aguas de arroyada». Rev. Las Ciencias, VI, 2, Madrid, pp. 337-358.

HESSINGER, E. (1949): «La distribución estacional de las precipitaciones en la Península Ibérica y sus causas». Estudios Geográficos, Madrid, pp. 59-129.

HUERTA, F. (1967): «El método de calcular el agua precipitada en un área de pocos datos». Boletín Mensual Climatológico, S.M.N., abril, Madrid, pp. 3-5.

HUERTA, F. (1969): La lluvia media de la España peninsular en el período 1931-1960. Notas de Meteorología Sinóptica, no 21, S.M.N., Mapa de isoyetas, período 1931-1960. Madrid.

IÑIGUEZ, F. (1909): «Las lluvias en nuestra Península». Anuario del Observatorio Central Meteorológico, Madrid, pp. 199-225.

J. S. E. (1966): «El régimen mensual, estacional y anual de lluvias en España». Calendario Meteorofenológico, S.M.N. Madrid, pp. 161-178.

JEFFERSON, M. (1932): «A rainfall map of the Iberian Peninsula». Geographical Review, XXII, Nueva York, pp. 678-680.

LAUTENSACH, H. (1934): Karte der durschnittlichen jährlichen Niederschlahöhe (1861-1900). In Klutes Hand. d. Geog. Wiss. Bd. Südost-u.Südeuropa. Wildpark-Postdam, Escala 1: 8.000.000, $451 \mathrm{pp}$. 
LAUTENSACH, H. (1951): «Die Niedersclagshöhen auf der Iberischen Halbinseln. Eine Geographische Studie». Petermanns Geog. Mitteilungen 3 Quart. 1 lám. mapa de isoyetas escala 1:1.500.000 y otra con mapas y gráficos, pp. 145-160.

LAUTENSACH, H. (1956): «El ritmo de las estaciones en la Península Ibérica». Est. Geog. Madrid, pp. 443-460.

LAUTENSACH, H \& MAYER, E. (1960): «Humidität und Aridität insbesondere auf der Iberischen Halbinseln». Petermanns Geog. Mitteilungen, 4 Quart.

LAUTENSACH, H. (1971): La precipitación en la Península Ibérica. S.M.N., Notas de Meteorología Sinóptica, no 25, marzo. Madrid.

LINES ESCARDO, A. (1970): The Climate of the Iberian Peninsula. Climates of northen and western Europe, C.C. Wallen (ed.), pp. 195-239. En World Survey of climatology, Vol. 5, H. E. Landsberg, Amsterdam.

LISO PUENTE, M. (1946): Actividades de la Estación de Pirineos II. 3. Sección de Climatología. Mapa de precipitación de los Pirineos españoles 1941/45. Escala 1:3.500.000.

LORENTE, J. Ma . (1951): «La varibilidad de las precipitaciones atmosféricas sobre España». II Congreso Nacional de Ingeniería. Tomo V, Madrid, pp. 429-434.

LORENTE, J. Mª (1955): «La variabilidad de las precipitaciones atmosféricas sobre España Peninsular». Rev. de Geofísica, XIV, Madrid, pp. 229-242.

LORENTE, J. Mª (1960): «Los problemas de la pluviometría en España». Rev. de Geofísica, XIX, Madrid, 171-181.

LORENTE, J. M ${ }^{\mathrm{a}}$. (1961): «La variabilidad de las precipitaciones atmosféricas sobre España peninsular durante los años 1947-48 hasta 1960-61». Rev. de Geofísica, XX, Madrid, pp. 229-245.

LORENTE, J. Mª (1973): «Precipitaciones acuosas sobre España Peninsular (en 10 metros cúbicos), acumuladas cada año de julio a junio siguiente». Calendario Meteorofenológico, S.M.N. Madrid, pp. $170-172$.

LORENZO PARDO, M. (1933): Plan general de Obras Públicas. Servicio Central Hidráulico. Madrid.

MASACHS ALAVEDRA, U. (1954): El clima. Las Aguas. En Geografía de España y Portugal, dirigido por D. Manuel de Terán, Tomo II, Ed. Montaner y Simón. Barcelona.

MARTIN VIDE, J. (1994): «Diez características de la pluviometría española decisivas en el control de la demanda y el uso del agua». Bol. de la Aso. Geog. Esp. n 18. Madrid, pp. 9-16.

MATEO GONZÁLEZ, P. (1954): «Determinación de los promedios pluviométricos en una estación con escaso número de datos». Boletín Mensual Climatológico, S.M.N., febrero pp. 2-3.

METEOROLOGICAL OFFICE (1943): Charts of rainfall over the Mediterranean region. M.O.M., Gran Bretaña. 441 págs.

MINISTERIO DE OBRAS PÚBLICAS (1942): Mapa pluviométrico de España y Portugal. Madrid.

MINISTERIO DE OBRAS PÚBLICAS (1969): Datos climáticos. Precipitaciones en períodos de 5 dias. Vol. I, II y III. Madrid.

MURRIEL FERNÁNDEZ, J. L. y MARTÍN ARANDA, J. (1973): «Observaciones sobre las características y distribución de lluvia en un área experimental». Rev. Urania, no 277-278.

PALOMARES CASADO, M. (1956): Las precipitaciones atmosféricas. Boletín Informativo de la Unión de Explosivos (Española), 56, noviembre, Madrid.

PERTIERRA, J. M. (1954): «Variación secular de la lluvia en España». Rev. Las Ciencias, XIX (3), Madrid, pp. 593-598.

S. M. N. (1942): Mapa pluviométrico de España (lluvias anuales medias: 1913-1932). Madrid.

S. M. N. (1943): Las series más largas de observaciones pluviométricas en la Península Ibérica. Serie D (Estadísticas), $\mathrm{n}^{\circ}$ 1, Madrid.

S. M. N. (1946): «El régimen anual de lluvias en la Península Ibérica». Calendario Meteorofenológico. Madrid, pp. 121-130.

S. M. N. (1965): Mapa pluviométrico de España (Lluvias medias anuales: 1931-1960). Madrid.

SEMMELHACH, W. (1910): Eiträge zur klimatogrephie von Norspanienn und Portugal. I - Die Niederschlag verjältnisse. Arch. Deustch. Seewarte. 33. 82 pp. Mapas de isoyetas anuales y estaciones del N y NW de la Península. Escala 1:4.000.000. 
SEMMELHACH, W. (1932): «Niederschlagkarte der Iberischen Halbinseln». Periode 1861-1900 ann. d. Hydr. u. Martti. meteorologie. 60, pp. 28-32. Mapa de isoyetas escala 1: 4.000.000.

TEMPERO (1960): «Estadística y clima. Precipitaciones atmosféricas en España». Tempero, abril. Zaragoza, pp. 35-39.

URIARTE HUMARA, E. (1960): «Lluvias y corrientes superficiales en España». Publication 51, International Association of Scientif Hydrology, pp. 601-615.

Dirección para correspondencia: José Jaime Capel Molina. Dpt ${ }^{\circ}$ de Geografía. Facultad de Letras, Universidad de Murcia. Apdo. 4021, 30080 - Murcia (España). 